\title{
Manuales y textos de psicofarmacología: una revisión
}

\section{Manuals and books of psychopharmacology: a review}

\author{
Andrés Molero Chamizo ${ }^{1}$ y G. Nathzidy Rivera Urbina \\ Facultad de Ciencias de la Educación, Campus El Carmen \\ Universidad de Huelva, España
}

(Recepción: Septiembre 2009 - Aceptación: Junio 2010)

\begin{abstract}
Resumen
En este trabajo se muestra una revisión de los principales y actuales textos, manuales y trabajos de Psicofarmacología publicados en lengua española, o traducidos a esta lengua. Métodos. Se realiza una revisión bibliográfica de aquellos materiales de Psicofarmacología que son relevantes en este campo de la ciencia. Resultados y Conclusiones. La revisión realizada resulta un material útil tanto para los profesionales relacionados con la Psicofarmacología, como para aquellos que se inicien en el conocimiento o en la investigación de las bases farmacológicas de nuestro comportamiento.
\end{abstract}

Palabras claves: Manuales; Psicofarmacología; Psicofármacos; Neurociencia; Psicopatologías.

\begin{abstract}
Introduction. This work shows a review of the main current books and manuals of Psychopharmacology published in Spanish, or translated into this language. Methods. A bibliographic review is made of those materials that are relevant in Psychopharmacology. Results and Conclusions. This review is a useful material for professionals involved in Psychopharmacology, and for those who are interested in the knowledge or research of the pharmacological basis of our behavior.
\end{abstract}

Key words: Manuals; Psychopharmacology; Drugs; Neurosciences; Psychopathology.

1 Correspondencia a: Andrés Molero Chamizo. Avda. Fuerzas Armadas s/n 21007 Huelva, Universidad de Huelva. Teléfono: 959-219503. E-mail: andres.molero@dpsi.uhu.es. 
El estudio de los sustratos y los mecanismos nerviosos responsables de nuestras cogniciones, emociones, motivaciones y comportamientos es un objetivo compartido por muchas disciplinas de la neurociencia. En particular, las diversas formas de estudio de los mecanismos farmacológicos de las sustancias psicoactivas y de sus efectos sobre el sistema nervioso y la conducta constituyen los aspectos esenciales de la psicofarmacología [Morales, 1990; Torres y Escarabajal, 2004, 2005], y conforman una parte importante de esta gran rama de la ciencia interesada en conocer la relación entre el sistema nervioso y sus funciones. La psicofarmacología es, pues, una disciplina de la neurociencia que estudia las bases biológicas del comportamiento. Y el método que utiliza la psicofarmacología para entender esta relación consiste en analizar los efectos de las sustancias farmacológicas sobre el sistema nervioso y sus funciones. Existen numerosos tratados, manuales, textos y artículos científicos que tratan alguno o algunos de los aspectos esenciales de la psicofarmacología. El objetivo de este trabajo es dar a conocer aquellos manuales y textos de psicofarmacología redactados en español que, por su relevancia en este campo, merecen ser destacados y que son utilizados con frecuencia en los ámbitos clínicos, docentes y de investigación.

Un libro con información muy elaborada y actualizada sobre psicofarmacología, y que es de gran utilidad en el estudio de esta disciplina, es el manual de Stephen M. Stahl (2002), Psicofarmacología esencial. Bases neurocientificas y aplicaciones clínicas. Este libro es un material de referencia en los estudios universitarios y recoge, por ejemplo, toda la información necesaria para la formación en la asignatura de psicofarmacología. En el estudio de esta disciplina se hace necesario conocer los diversos sistemas de neurotransmisión, así como los mecanismos de acción farmacológica de las sustancias psicoactivas y sus efectos conductuales. En este texto se puede encontrar información detallada de todos estos aspectos de la psicofarmacología. Existen dos ediciones del manual, una del año 1998 (Stahl, 1998), y la segunda del año 2002. Esta segunda edición resulta más completa que la anterior, y está compuesta de catorce capítulos, todos ellos de suma importancia para la formación en psicofarmacología. Así, en un primer capítulo se describen "Los principios de la neurotransmisión química", aspecto este esencial para entender la bioquímica del cerebro. En el segundo capítulo se describen "Los receptores y enzimas como objetivos de la acción de los fármacos", el cual se completa con el tercer capítulo titulado "Propiedades especiales de los receptores". Ya en el cuarto capítulo se hace una revisión de "La neurotransmisión química como mediadora de la acción de las enfermedades", el cual da paso a los siguientes capítulos dedicados a la psicofarmacología de los trastornos de la conducta. En particular, el capítulo cinco trata sobre la "Depresión y trastornos bipolares", el sexto sobre "Antidepresivos clásicos, inhibidores selectivos de la recaptación de serotonina e inhibidores de la recaptación noradrenérgica", el capítulo siete sobre "Nuevos antidepresivos y estabilizadores del estado de ánimo", el ocho sobre "ansiolíticos y sedanteshipnóticos", el nueve sobre "Tratamientos farmacológicos para el trastorno obsesivo-compulsivo, el trastorno de pánico y los trastornos fóbicos", el diez sobre "Psicosis y esquizofrenia", el once sobre "agentes antipsicóticos", el doce sobre "Potenciadotes cognitivos", el trece sobre "Psicofarmacología de la recompensa y sustancias de abuso", y el catorce sobre "Psicofarmacología sexualmente específica y relacionada con la función sexual". Como puede comprobarse, los contenidos de este texto recogen la información necesaria para una completa formación en psicofarmacología. En algunos capítulos aparecen expresiones de marcado carácter técnico, propias del ámbito profesional de esta disciplina, y explicaciones moleculares excesivamente detalladas, quizá, para un lector no muy formado en esta área. No obstante, en general, el libro resulta de gran utilidad para adentrarse en el campo de la psicofarmacología. Además, prácticamente todos los mecanismos de acción que se explican en el mismo están ilustrados y ejemplificados con notable maestría, lo cual resulta muy útil para todo aquel que esté interesado en adentrarse en el conocimiento de esta disciplina. Por tanto, resulta un texto muy recomendable para la formación básica en psicofarmacología.

Además del libro de Stahl (2002), existen varios manuales específicos y generales que tratan el campo de la psicofarmacología. Así, por ejemplo, también resulta muy recomendable para su estudio el texto traducido al español de Ronald W. Pies (2000), Manual de psicofarmacología básica. Éste es un texto menos extenso que el anteriormente citado. Sin embargo, trata sobre cuestiones esenciales de la psicofarmacología, que pueden complementar las explicaciones del anterior. Su perspectiva es 
fundamentalmente básica, si bien aparece información muy detallada de las distintas aplicaciones clínicas relacionadas con las sustancias psicoactivas que se describen. En este sentido, existe al final del capítulo un apartado dedicado monográficamente a la exposición de casos clínicos, y uno previo sobre preguntas y respuestas, en el que se puede encontrar abundante información acerca de cuestiones relacionadas con la psicofarmacoterapia. Los cuatro capítulos que preceden a estos dos últimos apartados tratan sobre la psicofarmacología de cuatro entidades clínicas bien definidas: la depresión, la psicosis, la ansiedad y los trastornos del estado de ánimo. En consecuencia, no resulta un texto tan detallado como el anteriormente citado, pero sí es un material directo, útil y práctico para el estudio de la bioquímica de estas psicopatologías.

Otro material importante para esta asignatura, escrito originariamente en español, es el texto Farmacología de la conducta. Manual básico para psicoterapeutas y clínicos, editado por G. Gómez-Jarabo (1999). En su introducción, Gómez-Jarabo expresa que el libro está "dirigido claramente" a estudiantes de psicología, si bien su propia organización le permite, en palabras del editor, ser un recurso útil en otras titulaciones. El texto está dividido en cuatro partes, con un total de veinte capítulos. El capítulo veinte recoge un índice de abreviaturas y de terminología y una relación alfabética de principios activos con acción psicofarmacológica, que resultan especialmente útiles tanto para su consulta teórica como para su aplicación práctica. En la primera y segunda parte del libro se hace una revisión del concepto de psicofarmacoterapia (capítulo uno), de la organización anatómica del sistema nervioso (capítulo dos), de la organización funcional del sistema nervioso (capítulo tres), de la fisiología y farmacología de la comunicación neuronal (capítulo cuatro), así como de los sistemas de neurotransmisión clásicos: la acetilcolina (capítulo cinco), las monoaminas (capítulo seis) y los aminoácidos, neuropéptidos y nucleósidos (capítulo siete). Ya en la tercera parte se describen los principales agentes psicoactivos de necesario conocimiento en psicofarmacología. Así, en el capítulo ocho se revisan los mecanismos de acción, la farmacocinética, las indicaciones y las interacciones farmacológicas de los sedantes, hipnóticos y somníferos. El capítulo nueve está dedicado por completo a la farmacocinética y farmacodinámica del alcohol, a sus efectos sobre el sistema nervioso y el metabolismo general, así como a los mecanismos de esta sustancia que inducen tolerancia y dependencia. El capítulo diez trata sobre la psicofarmacología de los estimulantes nerviosos y de las metilxantinas. En los capítulos once, doce y trece se repasa, respectivamente, la psicofarmacoterapia de tres clásicas entidades clínicas: la depresión, la ansiedad y las psicosis. En el capítulo catorce se describen las propiedades y los mecanismos de acción de los reguladores o estabilizadores del ánimo, y en el capítulo quince se detalla la psicofarmacología de los anticonvulsivos. El capítulo 16 está dedicado a las sustancias con propiedades vasodilatadoras, psicoenergizantes y nootrópicas (término acuñado para referirse a aquellos agentes potenciadores de la cognición), y a su aplicación terapéutica en la sintomatología cognitiva de la vejez y la demencia. En el capítulo diecisiete se revisan las principales sustancias psicodélicas y drogas de diseño, y en el dieciocho se describen los mecanismos del dolor y las propiedades antinociceptivas de los analgésicos no opiáceos. Por su parte, los analgésicos opiáceos y sus mecanismos de acción son tratados en el tema diecinueve. Finalmente, la cuarta y última parte incluye el capítulo veinte, en el que aparece un índice de abreviaturas y de terminología y una relación alfabética de principios activos con acción psicofarmacológica, que resultan de gran valor tanto para su consulta teórica como para su aplicación práctica en la clínica.

Además de estos textos señalados, en los últimos ocho años han aparecido diversas publicaciones relacionadas con la psicofarmacología, muy completas y actualizadas. Cabe destacar los libros Psicofarmacología para psicólogos (Bravo, 2002), Farmacología de la conducta (FernándezTeruel, 2008), Diagnóstico diferencial y racionalización del tratamiento psicofarmacológico (Pichot 2001), Psicofarmacología y otras terapias biológicas (Guindeo y Rios, 2004), y El tratamiento farmacológico en psiquiatría: indicaciones, esquemas terapéuticos y elementos para su aplicación racional (Wikinski, 2005).

Como ya se ha indicado, estos libros descritos son bastante completos para la formación en esta disciplina. Existe un manual de psicofarmacología algo menos denso, con un formato de bolsillo, pero que contiene información básica, química y clínica de una enorme cantidad de sustancias 
psicoactivas. Se trata del Manual de psicofarmacología, editado por Salazar, Peralta y Pastor (2006), que en realidad es una reedición resumida y revisada del texto matriz de los mismos editores y autores titulado Tratado de psicofarmacología: bases y aplicación clínica (Salazar et al., 2005a). $\mathrm{Su}$ contenido está organizado en tres secciones. En la primera sección, que incluye los capítulos del uno al tres, se describen aspectos relevantes de la psicofarmacoterapia en distintos grupos de pacientes y en situaciones específicas. En la sección dos (que contiene los capítulos que van del cuatro al treinta y nueve) se exponen los mecanismos farmacológicos y las indicaciones clínicas de numerosas sustancias psicotrópicas, e incluso de varias hormonas, prostaglandinas y vitaminas. En la tercera y última sección aparece un índice por principios activos y por nombre comercial de éstos en España, notablemente práctico y de gran utilidad para los profesionales de la salud. Estos editores crearon, además, una guía sobre su propio tratado anteriormente mencionado, para su mejor manejo y utilización. Se trata de la Guía de estudio del tratado de Psicofarmacología. Autoevaluación razonada (Salazar et al., 2005b).

En cuanto a la perspectiva puramente clínica, pueden citarse tres textos importantes con esta orientación, uno publicado a finales de los años ochenta y otros dos mucho más recientes. Tales libros son Psicofarmacología clínica (Bueno et al., 1986), Manual de psicofarmacología clínica (Silva Ibarra, 2003) y Manual de psicofarmacología clínica (Schatzberg, 2005), éste último traducido del inglés. Por otra parte, para aquellos que estén interesados en los orígenes y la historia de la psicofarmacología, existe un tratado de tres tomos con ingente información sobre la génesis de esta disciplina de la ciencia. Se trata de los manuales Historia de la psicofarmacologia. Tomo I: de los orígenes a la medicina científica: sobre los pilares biológicos del nacimiento de la psicofarmacología, Tomo II: la revolución de la psicofarmacología: sobre el descubrimiento y desarrollo de los psicofármacos, Tomo III: la consolidación de la psicofarmacología como disciplina cientifica: aspectos éticos-legales y perspectivas de futuro (López y Álamo, 2007).

Todos estos libros pueden considerarse tratados generales de psicofarmacología. En lengua castellana existe además un texto traducido del inglés (por José Luis Cantero y Mercedes Atienza), que trata específicamente de los mecanismos del sueño, de su bioquímica y de la psicofarmacoterapia y psicoterapia de los trastornos del sueño. La farmacia de los sueños: la alteración química de los estados de consciencia (Hobson, 2003) es un texto relativamente breve, que puede complementar los conocimientos generales adquiridos en psicofarmacología, y que puede ayudar a entender este apasionante campo de la neurociencia en uno de sus múltiples campos de actuación: los trastornos de la consciencia y del sueño. Su contenido se organiza en seis partes. En la primera de ellas se incluyen los capítulos uno, dos y tres, que tratan sobre "El campo de estudio y la configuración de los estados de consciencia". La segunda parte, que lleva por título "Más allá del psicoanálisis: hacia una teoría neurodinámica de los estados de consciencia", contiene los capítulos cuatro y cinco. Los capítulos que van del seis al nueve se incluyen en el tercer apartado, que está dedicado a las "Alteraciones normales y anormales de la consciencia". La cuarta parte la forman los capítulos diez, once y doce, que tratan sobre la "Farmacia médica". En la quinta parte se exponen algunos aspectos de lo que el autor ha denominado la "Farmacia recreativa", a lo largo de los capítulos trece, catorce y quince. Y en la sexta y última parte (que el autor titula "La farmacia psicológica"), en el capítulo dieciséis, se abordan cuestiones relevantes para el tratamiento y la psicofarmacoterapia, como la vinculación terapéutica, la cooperación o resistencia en el proceso terapéutico, etc. Por último, al final del texto puede encontrarse un índice temático que puede ayudar a localizar con gran facilidad información acerca de diversos conceptos y sustancias psicoactivas, particularmente a los que se inicien en el estudio de la psicofarmacología o de algún aspecto de ésta.

Otro material que centra su atención en una dimensión concreta de la psicofarmacología es el libro Psicofarmacología de los trastornos cognoscitivos y psiquiátricos en el anciano (Wheatley y Smith, 2001). Su contenido resulta adecuado para profundizar en el conocimiento de la psicofarmacoterapia, aunque en una población especial como son los pacientes geriátricos. La terapia farmacológica de los trastornos de memoria y del estado de ánimo (frecuentes en la población anciana, y particularmente en sujetos con algún tipo de demencia) es el objetivo principal de este texto. El libro está estructurado en tres apartados. En el primero de ellos, que lleva por título 
“Conceptos básicos", se describen las causas del envejecimiento (capítulo uno), la farmacocinética de los fármacos psicotropos en los ancianos (capítulo dos), y los efectos adversos de los fármacos psicotropos (capítulo tres). El segundo apartado se centra en los "Trastornos cognoscitivos", y en él aparece información resumida sobre los sustratos neuroquímicos de la demencia (capítulo cuatro), sobre las implicaciones neuropatológicas para la terapia (capítulo cinco), sobre los fármacos para la enfermedad de Alzheimer (capítulo seis), y sobre la evaluación de la memoria (capítulo siete). Por último, el tercer apartado versa sobre los "Trastornos psiquiátricos". El capítulo ocho de este apartado se centra en la ansiedad en ancianos, el nueve lo hace en el tratamiento de la depresión en edades avanzadas, el diez trata sobre trastornos físicos y enfermedad psiquiátrica, el once describe los trastornos del sueño, el doce muestra algunos aspectos de la psicosis paranoide en la vejez, y el capítulo trece está dedicado a las conductas perturbadoras en el anciano. Al final del apartado tres aparece un índice alfabético de materias, que remite a las páginas del texto en las que puede hallarse información de algunos términos para su consulta.

Otros textos y artículos de psicofarmacología sobre poblaciones especiales y grupos de consumo son Psicofarmacología del niño y del adolescente (Mardomingo, 1997), Psicofarmacología clínica de la infancia y la adolescencia (Toro, 1998), Medicina del sueño. Enfoque multidisciplinario (Velayos, 2009), Bases neurológicas de la adicción a la cocaína (Pulvirenti y Koob, 1996), Drogas y cerebro (Snyder, 1992), Psicofarmacología de la nicotina y conducta adictiva (Molero y Muñoz, 2005), Neurobiología de la drogadicción (Ramos Atance, 1993), Drogodependencias. Farmacología. Patología. Psicología. Legislación (Lorenzo et al., 2003), Cerebro y adicción: una guía comprensiva (Tirapu, 2004), La historia del LSD (Hofmann, 2001), Marihuana: conocimiento científico actual (Iversen, 2001), Cannabinoides: aspectos psiquiátricos y bioquímicos (Solé y Ramos, 2001).

Merece igualmente ser destacado el libro traducido al castellano Psicofarmacología esencial de antipsicóticos y estabilizadores del estado de ánimo (Stahl, 2003). En él se revisan los aspectos psicofarmacológicos de los neurolépticos y de otros agentes con propiedades antipsicóticas y reguladoras del humor. En particular, se realiza una revisión exhaustiva de los efectos moduladores sobre los receptores dopaminérgicos de la nueva generación de antipsicóticos atípicos, tales como el aripiprazol o la ziprasidona. Además de éste, otros materiales (libros o artículos científicos) dedicados al tratamiento y a la farmacología de algún trastorno psiquiátrico concreto, o grupo de trastornos, son Psicofarmacología esencial de la depresión y trastorno bipolar (Stahl, 2002), Éxitos y paradojas de los antidepresivos (Ammar y Jouvent, 1995), Novedades en el tratamiento del trastorno bipolar (Vieta, 2007), Perspectivas neurobiológicas del trastorno por déficit de atención con hiperactividad (Cabanyes y Polaino, 1992), ¿Se puede prescindir de los ansiolíticos? (Escande, 1995).

Además de estos textos y manuales, los profesionales de la salud y el estudiante de psicofarmacología tienen a su alcance varias guías de terapia farmacológica general, donde pueden hallar diversos aspectos técnicos y clínicos de las sustancias psicoactivas, sus principios activos y sus indicaciones terapéuticas. El manual Medimecum. Guía de terapia farmacológica (Villa, 2003) es una de estas guías escritas en español y enfocada a la farmacología en nuestro país. Una de sus secciones está dedicada a la farmacología del sistema nervioso y a las pautas de prescripción de sustancias psicotrópicas.

Quienes estén interesados en el campo de la psicofarmacología también pueden consultar algunos de los manuales y textos clásicos de psicobiología, neurobiología, neurología, farmacología, y neurociencia en general, aunque éstos sean menos específicos. Algunos de estos textos en los que se trata de manera general conceptos de neurotransmisión y psicofarmacología son Fisiología de la conducta (Carlson, 2006), Manual de neurociencia (Delgado et al., 1998), Neurociencia y conducta (Kandel et al., 1996), Principios de neurociencia (Kandel et al., 2000), Biopsicología (Pinel, 2007), Fundamentos biológicos de la conducta (Del abril et al., 2001), Neurociencia (Purves et al., 2007), Neurociencia aplicada (Cardinali, 2007), Psicología biológica (Rosenzweig y Leiman 2002), Psicobiología (Rosenzweig et al. 2005), Harrison. Neurología en medicina clínica (Hauser, 2006), Principios de neurología, de Adams y Victor (Ropper y Brown, 2007), Avances en psiquiatría biológica 
(Mendlewicz, 1992), Neuroquímica (Bradford, 1989), Neurociencia: explorando el cerebro (Bear et al., 2002), Farmacología clínica para profesionales de la salud (Azanza, 2004), Farmacología Humana (Flórez, 2004), Farmacología (Rang y Dale, 2000), Farmacología fundamental (Velasco, 2004), Farmacología básica y clínica (Velázquez y Lorenzo, 2005).

Aparte de todos los materiales en español hasta aquí citados, existen algunos otros en inglés relacionados con psicofarmacología, no traducidos a nuestro idioma, que merecen ser resaltados. Entre ellos destacan los manuales Principles of neuropsychopharmacology (Feldman et al., 1997), Psychopharmacology. The fourth generation of progress (Bloon y Kupfer, 1995), y Neurobiology of addiction (Koob, 2006), o varios artículos científicos recientes (Aghajanian, 2009; Bradshaw, 2002; Porter y Prus, 2009; Silva, 2009).

En definitiva, estos son algunos de los materiales disponibles tanto para profesionales como para estudiantes interesados en el campo de la psicofarmacología. No es una revisión exhaustiva, pero puede servir para disponer de importantes recursos bibliográficos que tratan sobre aspectos esenciales de esta área de la neurociencia.

\section{Referencias}

Aghajanian, G.K. (2009). Modeling "psychosis" in vitro by inducing disordered neuronal network activity in cortical brain slices. Psychopharmacology, online.

Ammar, S. y Jouvent, R. (1995). Éxitos y paradojas de los antidepresivos. Mundo Científico, 163, 1072-1076.

Azanza Perea, J. R. (2004). Farmacología clínica para profesionales de la salud. Pamplona: Eunate.

Bear, M.F., Connors, B.W. y Paradiso, M.A. (2002) Neurociencia: explorando el cerebro. Barcelona. Masson.

Bloon, F.E. y Kupfer, D.J. (Eds.) (1995). Psychopharmacology. The fourth generation of progress. Raven Press.

Bradford (1989). Neuroquímica. Labor.

Bradshaw, J.L. (2002). Developmental disorders of the frontostrial systems. Psychology Press.

Bravo, M.F. (2002). Psicofarmacología para psicólogos. Madrid: Síntesis.

Bueno, J.A., Sabanes, F., Salvador, L. y Gascón, J. (1986). Psicofarmacología clínica. Barcelona: Salvat.

Cabanyes Truffino, J. y Polaino-Lorente. A. (1992). Perspectivas neurobiológicas del trastorno por déficit de atención con hiperactividad. Medicina Clínica, 98, 591-594.

Cardinali, D.P. (2007). Neurociencia aplicada. Buenos Aires: Editorial Médica Panamericana.

Carlson, N.R. (2006) Fisiología de la conducta. $8^{\text {a }}$ edición. Pearson Educación. Madrid.

Del abril Alonso, A., Ambrosio Flores, E., De blas Calleja, M.R., Caminero Gómez, A.A., García Lecumberri, C., De pablo González, J.M. y Sandoval Valdemoro, E. (2001). Fundamentos biológicos de la conducta. $2^{\text {a }}$ edición. Sanz y Torres.

Delgado, J.M., Ferrús, A., Mora, F. y Rubia, F.J. (1998). Manual de neurociencia. Madrid: Editorial Síntesis.

Escande, M. (1995) ¿Se puede prescindir de los ansiolíticos? Mundo Científico, 163, 1067-1071.

Feldman, R.S., Meyer, J.S. y Quenzer, L.F. (1997). Principles of neuropsychopharmacology.

Sunderland, Mass.: Sinauer Associates. 
Fernández-Teruel, A. (2008). Farmacología de la conducta. Barcelona: Servicio de Publicaciones de la Universidad de Barcelona.

Flórez, J. (2004). Farmacología Humana. Barcelona: Masson.

Gómez-Jarabo, G. (Ed.) (1999). Farmacología de la conducta: manual básico para psicoterapeutas y clínicos. Síntesis Psicología.

Guindeo, J. y Rios, B. (Eds.) (2004). Psicofarmacología y otras terapias biológicas. Editorial Lilly.

Hauser, S.L. (2006). Harrison. Neurología en medicina clínica. McGraw-Hill.

Hobson, J.A. (2003). La farmacia de los sueños: la alteración química de los estados de consciencia. Barcelona: Ariel Neurociencia.

Hofmann, A. (2001). La historia del LSD. Barcelona: Gedisa.

Iversen, L.L. (2001). Marihuana: conocimiento cientifico actual. Barcelona: Ariel.

Kandel, E.R., Jessell, T.M. y Schwartz, J.H. (1996). Neurociencia y conducta. Editorial Prentice Hall.

Kandel, E.R., Jessell, T.M. y Schwartz, J.H. (2000). Principios de neurociencia. McGraw-Hill.

Koob, G. F. (2006). Neurobiology of addiction. Amsterdam: Elsevier.

López, F. y Álamo, C. (2007). Historia de la psicofarmacología. Tomo I: de los orígenes a la medicina científica: sobre los pilares biológicos del nacimiento de la psicofarmacología. Madrid: Editorial Médica Panamericana.

López, F. y Álamo, C. (2007). Historia de la psicofarmacología. Tomo II: la revolución de la psicofarmacología: sobre el descubrimiento y desarrollo de los psicofármacos. Madrid: Editorial Médica Panamericana.

López, F. y Álamo, C. (2007). Historia de la psicofarmacología. Tomo III: la consolidación de la psicofarmacología como disciplina científica: aspectos éticos-legales y perspectivas de futuro. Madrid: Editorial Médica Panamericana.

Lorenzo, P., Ladero, J.M., Leza, J.C. y Lizasoain, I. (Eds.) (2003). Drogodependencias. Farmacología. Patología. Psicología. Legislación. Madrid: Panamericana.

Mardomingo, M. J. (1997). Psicofarmacología del niño y del adolescente. Madrid: Díaz de Santos.

Mendlewicz (Ed.) (1992). Avances en psiquiatría biológica. Barcelona: Masson.

Molero, A. y Muñoz, J. E. (2005). Psicofarmacología de la nicotina y conducta adictiva. Trastornos adictivos, 7, 137-152.

Morales, A. (1990). Psicofarmacología: Multiplicidad de enfoques. Revista de Psicología General y Aplicada, 43, 121-126.

Pichot, P. (2001). Diagnóstico diferencial y racionalización del tratamiento psicofarmacológico. Madrid: Aula Médica Ediciones.

Pies, R.W. (2000). Manual de psicofarmacología básica. Barcelona: Masson.

Pinel, J.P. (2007). Biopsicología. $6^{a}$ edición. Prentice Hall.

Porter, J.H. y Prus, A.J. (2009). Drug discrimination: 30 years of progress. Psychopharmacology, online.

Pulvirenti, L. y Koob, G.F. (1996). Bases neurológicas de la adicción a la cocaína. Investigación y Ciencia, 238, 48-55. 
Purves, D., Augustine, G.J., Fitzpatrick, D., Hall, W.C., LaMantia, A.S., McNamara, J.O. y Willians, S.M. (2007). Neurociencia. Madrid: Editorial Médica Panamericana.

Ramos Atance, J.A. (1993). Neurobiología de la drogadicción. Eudema, Madrid.

Rang, H.P. y Dale, M.M. (2000). Farmacología. Madrid: Harcourt Brace de España, S.A.

Ropper, A.H. y Brown, R.H. (2007). Principios de neurología, de Adams y Victor. McGraw-Hill.

Rosenzweig, M.R. y Leiman, A.L (2002). Psicología biológica. McGraw-Hill.

Rosenzweig, M.R., Breedlove, S. M. y Watson, N.V. (2005). Psicobiología. Barcelona: Ariel.

Salazar, M., Peralta, C. y Pastor, J. (Eds.) (2005a). Tratado de psicofarmacología: bases y aplicación clínica. Madrid: Editorial Médica Panamericana.

Salazar, M., Peralta, C. y Pastor, J. (Eds.) (2005b). Guía de estudio del tratado de Psicofarmacología. Autoevaluación razonada. Editorial Médica Panamericana.

Salazar, M., Peralta, C. y Pastor, J. (Eds.) (2006). Manual de psicofarmacología. Madrid: Editorial Médica Panamericana.

Schatzberg, Alan F. (2005). Manual de psicofarmacología clínica. Barcelona: Ars Medica.

Silva Ibarra, H. (Ed.) (2003). Manual de psicofarmacología clínica. Santiago, Chile: Mediterráneo.

Silva, R. (2009). Psychopharmacology news. Journal of Child and Adolescent Psychopharmacology, 19, 91-91.

Snyder, S. H. (1992). Drogas y cerebro. Prensa Cientifica.

Solé, J.R. y Ramos, J.A. (Eds.) (2001). Cannabinoides: aspectos psiquiátricos y bioquímicos. Madrid: Ediciones Díaz de Santos.

Stahl, S.M. (1998). Psicofarmacología esencial: bases neurocientificas y aplicaciones clínicas. $1^{\mathrm{a}}$ Edición. Barcelona: Ariel Neurociencia.

Stahl, S. M. (2002). Psicofarmacología esencial. Bases neurocientificas y aplicaciones clínicas. $2^{\mathrm{a}}$ edición actualizada. Ariel Neurociencia.

Stahl, S. M. (2002). Psicofarmacología esencial de la depresión y trastorno bipolar. Barcelona: Ariel Neurociencia.

Stahl, S.M. (2003). Psicofarmacología esencial de antipsicóticos y estabilizadores del estado de ánimo. Barcelona: Ariel Neurociencia.

Tirapu Ustárroz, J. (2004). Cerebro y adicción: una guía comprensiva. Pamplona: Gobierno de Navarra, Departamento de Salud.

Toro, J. (Ed.) (1998). Psicofarmacología clínica de la infancia y la adolescencia. Barcelona: Masson.

Torres, C. y Escarabajal, M.D. (2004). Psicofarmacología: un enfoque psicobiológico. Psiquiatría Biológica, 11, 186-195.

Torres, C. y Escarabajal, M.D. (2005). Psicofarmacología: una aproximación histórica. Anales de Psicología, 5, 199-212.

Velasco, A. (2004). Farmacología fundamental Madrid: McGraw-Hill Interamericana.

Velayos, J.L. (2009). Medicina del sueño. Enfoque multidisciplinario. $1^{\text {a }}$ Edición. Madrid: Editorial Médica Panamericana.

Velázquez y Lorenzo (2005). Farmacología básica y clínica. Madrid: Editorial Médica Panamericana. 
Vieta, E. (2007). Novedades en el tratamiento del trastorno bipolar. $2^{\text {a }}$ Edición. Madrid: Editorial Médica Panamericana.

Villa, L.F. (2003). Medimecum. Guía de terapia farmacológica. Adis Internacional Ltd.

Wheatley, D. y Smith, D. (2001). Psicofarmacología de los trastornos cognoscitivos y psiquiátricos en el anciano. Barcelona: Masson.

Wikinski, S.(2005). El tratamientofarmacológicoen psiquiatría: indicaciones, esquemasterapéuticos y elementos para su aplicación racional. Buenos Aires: Editorial Médica Panamericana. 
